\title{
Diabetes and Cannabis: Myth or Reality
}

\section{María Luz Gunturiz Albarracín* and Pablo Enrique Chaparro}

Specialized professional, Public Health Research Division, National Institute of Health, Avenue Street 26 No 51-20 CAN, Bogotá, D.C., Colombia.
*Correspondence:

Maria Luz Gunturiz A. BsC, PhD, Specialized professional, Public Health Research Division, National Institute of Health, Colombia, Tel: +57(1) 2207700 Ext 1629; E-mail: mgunturiz@ins.gov.co.

Received: 19 December 2017; Accepted: 12 January 2018

Citation: María Luz Gunturiz Albarracín, Pablo Enrique Chaparro. Diabetes and Cannabis: Myth or Reality. Cardiol Vasc Res. 2018; 2(1); 1-6.

\begin{abstract}
Medical marijuana is one of the fastest growing industries in the United States and in developing countries such as Colombia, but research into the benefits of the plant is a global issue. Although there are studies that present evidence that people who take or smoke it, may have a lower susceptibility to diabetes due to metabolic acceleration caused by the plant in the body, with this review we intend to show that the evidence obtained is not is still enough to think that marijuana could be a treatment for diabetes or other non-transmissible chronic diseases. This presumption is due to the lack of knowledge of its adverse effects and the consequences of cannabis use on the severity of these pathologies. Additionally, more clinical studies are required to demonstrate that marijuana activates the balance between metabolism and glucose and therefore demonstrate that this "medicinal" plant can help patients who already have the disease to decrease their dependence on insulin.
\end{abstract}

Whether or not cannabis use may increase or decrease the risk of diabetes is not completely clear.

Keywords

Diabetes, Cannabis, Marijuana, Cannabinoids.

\section{Abbreviations}

DM1: Diabetes mellitus type 1, DM2: Diabetes mellitus type 2, THC: Delta 9-tetrahydrocannabinol, CBD: Cannabidiol, HDL-C: High-density lipoprotein C, NOD: non-obese diabetic, TNF- $\alpha$ : Tumor necrosis factor, NF- $\kappa \beta$ : Nuclear factor $k \beta$, MAPKs: Mitogen-activated protein kinase, PPAR: Peroxisome proliferatoractivated receptor.

\section{Introduction}

Cannabis is among the most widely used illicit drugs with an estimated 180 million people aged 15-64 years using it for nonmedical purposes [1]. In recent decades, the use of cannabis has been growing much more rapidly than the use of other substances, and the most rapid growth since 1960 has occurred in high-income countries [1]. The current wave of decriminalization may lead to more widespread use, and it is important to address the potential cannabis-associated adverse health effects that may begin to occur in the population at a greater frequency [2]. Previous studies have linked cannabis use to a variety of psychiatric and somatic disease including anxiety [3], schizophrenia [4,5], depression, dependence [6,7], chronic bronchitis [8], respiratory infections [9], lung cancer [10], myocardial infarction [2], and stroke [11,12].

Whether or not cannabis use may increase or decrease the risk of developing chronic noncommunicable diseases including type 2 diabetes (DM2) is still unresolved. The question is rarely studied and results are conflicting. The most well-established risk factors for DM2 are overweight and obesity, physical inactivity, unhealthy food intake, increasing age, smoking, high alcohol intake, genetics, and a family history of diabetes [13-15]. Therefore, while the association between several life-style factors and DM2 is established, possible associations between cannabis use and this disease remain to be clarified [12].

Composition of the plant and potential effects

The two main components in the cannabis plant are cannabidiol (CBD) and the delta 9-tetrahydrocannabinol (THC), with seemingly different characteristics. Both CBD and THC belong to 
a class of compounds known as cannabinoids, which are chemical components that act on cannabinoid receptors in cells that modulate neurotransmitter release in the brain [16].

The cannabinoids, (e.g. the most characterized active ingredient, the THC) exert significant analgesic effects by activating the classical CB1 receptors located in the central nervous system and inflammatory properties through the activation of cannabinoid CB2 receptors in immune cells. However, the main limitation of the therapeutic usefulness of these compounds is the development of CB1-dependent psychoactive effects centrally mediated [17].

Cannabidiol, a nonpsychoactive component of marijuana, has been shown to exert anti-inflammatory and antioxidant affects both in vitro and in various preclinical models of neurodegeneration and inflammatory disorders, independent from classical CB1 and CB2 receptors [18]. Furthermore, $\mathrm{CBD}$ has recently been reported to lower the incidence of diabetes in non-obese diabetic mice [19] and to preserve the blood-retinal barrier in experimental diabetes $[20,21]$.

\section{Cannabis and Diabetes}

Currently there are not many clinical studies on the use of cannabis for the treatment of diabetes, but there have been published a few preclinical studies that indicate that cannabinoids can modify the progression of the disease and provide relief of some symptoms $[22,23]$. A 2006, a study reported that injections of $5 \mathrm{mg}$ per day of non-psychoactive cannabinoid CBD significantly reduced the incidence of diabetes in mice. The researchers reported that $86 \%$ of control mice not treated in the study developed diabetes. In contrast, only $30 \%$ of mice treated with CBD developed the disease [24]. In another study conducted by the same researchers, it was reported that all control mice developed diabetes in a mean of 17 weeks (range 15 to 20 weeks), while the majority (60\%) of mice treated with CBD remained without diabetes at 26 weeks $[24,25]$.

Activation of $\mathrm{CB} 1$ receptors has been shown to promote food craving and hyperphagia also in humans [26]. This led to the development of endocannabinoid receptor antagonists as therapy for obesity and DM2 [27]. However, these drugs were withdrawn early from the market due to severe psychiatric adverse effects.

Marijuana use is associated with an acute increase in caloric intake, and people who smoke marijuana have higher average caloric intake levels than nonusers [28-30]. Despite these associations with increased caloric intake, marijuana use has been associated with lower body mass index (BMI) and a lower prevalence of obesity and diabetes mellitus [29,31,32]. The mechanisms underlying this paradox have not been determined, and the impact of regular marijuana use on insulin resistance and cardiometabolic risk factors remains unknown.

Although not completely elucidated, the mechanisms by which cannabinoids affect peripheral metabolism via these receptors have been studied previously y; the cannabinoid type 1 receptor antagonist, rimonabant, was found to improve insulin sensitivity in wild-type mice, but not in adiponectin knockout mice, suggesting that adiponectin at least partially mediates the improvement in insulin sensitivity; adiponectin has been reported to improve insulin sensitivity. This rimonabant-induced improvement in insulin resistance has been confirmed in human studies [33-35].

Furthermore, in a randomized clinical trial, rimonabant was significantly associated with an increase in plasma adiponectin levels, as well as weight loss and a reduction in waist circumference [36]. Cannabis itself, when administered to obese rats, was associated with weight reduction and an increase in the weight of pancreata, implying beta-cell protection [37]. In addition, cannabinoid type 1 knockout mice are resistant to dietinduced obesity, suggesting that the role of this receptor is central in the metabolic processes leading to obesity [38]. Given that 2 of the main active phytocannabinoids in marijuana, (-)-trans-D9 -tetrahydrocannabinol and cannabidiol, are classified as partial agonists and antagonists, respectively, and are thus capable of producing antagonistic effects at the cannabinoid receptors [39].

Animal studies have shown that cannabinoids, primarily $\mathrm{THC}$, stimulate appetite through activation of certain $\mathrm{CB} 1$ receptors and may play a role in compulsive eating behavior [40,41] while others have reported a significant reduction, or delayed onset, in the development of DM2 in CBD treated mice $[19,24]$. There is, however, little research evidence to support this direct effect of cannabis on humans.

Another independent observational study published in 2013 reported that subjects who used cannabis have favorable rates related to diabetic control compared to those who do not have a history of marijuana use. Researchers evaluated the relationship between marijuana use and fasting insulin, glucose and insulin resistance in a sample of 4,657 male subjects, concluding that those individuals who reported having used marijuana in the last month had lower fasting insulin levels and of insulin resistance, as well as the smaller waist circumference and higher levels of high density lipoprotein or cholesterol, HDL-C. On the other hand, these associations were milder among those who reported using marijuana at least once (not in the last 30 days), suggesting that the impact of marijuana use on insulin and insulin resistance exists during periods of recent use. Collectively, these results, strongly suggest that it may have great therapeutic potential in the treatment of diabetic complications, and perhaps other cardiovascular disorders, by attenuating oxidative/nitrosative stress, inflammation, cell death and fibrosis [39,31].

One cross-sectional study from the US showed that previous, current light and heavy cannabis users had relatively lower odds of diabetes compared with nonusers [32]. It has been suggested that cannabis use may lower the risk of diabetes by lowering fasting insulin levels, as well as the risk of insulin resistance [32,39]. Despite the fact that the use of cannabis often is associated with a unfavorable life-style, such as higher intake of calories, a higher tobacco smoking rate, higher consumption of alcohol, and illicit 
drugs [29], studies have also shown that cannabis use may lower body mass index (BMI) [42] and reduce the prevalence of obesity [31]. Moreover, a recent meta-analysis including eight separate US samples reported that active cannabis use was inversely associated with type 2 diabetes [43]. However, the included samples were all from cross-sectional studies and, as stressed by the authors, more research is needed $[12,43]$.

There are several studies that address the issue of marijuanadiabetes that are contradictory. While the investigations of Rajavashisth et al. in 2012, Sidney and Dagogo-Jack in 2016, suggest association between the use of cannabis and the reduction of the prevalence of diabetes mellitus [44,45], Bancks et al. in 2015 studying a cohort of healthy men and women reported that marijuana use is associated with a higher prevalence of prediabetes in young adults and middle-aged adults but found no association with diabetes for this same study population [47]. On the other hand, in a review article carried out by Sidney in 2016, it was pointed out that the studies included in his analysis showed a low probability of association between the use of marijuana and the prevalence of diabetes [45].

Neuropathy is the most common complication of diabetes and it is still considered to be relatively refractory to most of the analgesics. Studies on painful Diabetic Neuropathy, over time have also yielded variable results. Comelli et al. in 2009 in an experimental study in which they administered cannabis extract to rats that were induced by diabetes, found beneficial effects for the discomfort [48]. Selvarajah et al. in 2010 in an experimental study in patients with painful diabetic neuropathy, evaluated the efficacy of cannabis, administered sublingually, demonstrating that it was not more effective than placebo [49]. On the other hand, Wallace et al, in 2016, in an experimental study in patients with painful diabetic neuropathy evaluated their potential benefit, administered cannabis by inhalation and demonstrated that it was not effective [50].

Cannabinoids have also been shown to relieve neuropathic pain associated with the disease. Studies published in 2004 reported that mice administered with an agonist (activator) of cannabinoid receptors experienced a reduction in tactile allodynia (pain resulting from non-detrimental stimuli to the skin) related to diabetes compared with untreated controls, suggesting that "cannabinoids have a potential beneficial effect on experimental diabetic neuropathic pain" [51,52].

Among the possible explanations for these contradictory results is the exclusion of studies of unhealthy individuals who frequently use marijuana, which may lead to underestimation of cases of diabetes in marijuana users; people with diabetes who abstain from consuming marijuana in their old age; the same awareness about health status [44,53], how surveys are applied and how information is obtained, among others [47].

The powerful anti-inflammatory effect of $\mathrm{CBD}$, with reduction of cytokines production (IFN- $\gamma$ and TNF- $\alpha$ ) and inhibition of $\mathrm{T}$ cell proliferation observed in experimental arthritis, [54] led to investigation of the possible CBD effects on others diseases as the DM1 that is a disease that results in the destruction of insulin-producing pancreatic $\beta$ cells. The primary lesion of DM1 is an inflammation of the islands of Langerhans, during which leukocytes and lymphocytes in particular, surround and infiltrate the islets. Some studies have shown that CBD treatment of nonobese diabetic (NOD) mice before the development of the disease reduced its incidence from $86 \%$ in the non-treated control mice to $30 \%$ in CBD-treated mice. Additionally, CBD treatment resulted in important reduction of plasma levels of the pro-inflammatory cytokines, IFN- $\gamma$ and TNF- $\alpha$ and the histological examination of the pancreatic islets of CBD-treated mice revealed significant reduction of the inflammation [24]. It was also observed that administration of CBD to 11-14 week old female NOD mice, which were either in a latent diabetes stage or had initial symptoms of diabetes, ameliorated the manifestations of the disease. In addition, the level of the pro-inflammatory cytokine IL-12 produced by splenocytes was significantly reduced, whereas the level of the anti-inflammatory IL-10 was significantly elevated after CBD treatment. 98 This data have suggested that CBD can possibly be used as a therapeutic agent for the treatment of DM1.

History of research on cannabidiol CBD has also been proven useful for possible complications of diabetes. The majority of diabetic complications are associated with pathophysiological alterations in the vasculature. Microvascular complications involve retinopathy and nephropathy while the atherosclerosis is the most common macrovascular complication of diabetes. The protective effects of CBD were studied in experimental diabetes induced by streptozotocin in rats. CBD treatment prevented retinal cell death and vascular hyperpermeability in the diabetic retina.

In addition, it significantly reduced oxidative stress, decreased the levels of TNF- $\alpha$, vascular endothelial growth factor, and intercellular adhesion-molecule [20]. It has also been suggested that $\mathrm{CBD}$ has significant therapeutic benefits against other diabetic complications and atherosclerosis, since it attenuated several effects of high glucose, including the disruption of the endothelial function $[55,56]$.

Cardiovascular complications are the main cause of morbidity and mortality in diabetic patients. For example, the diabetic cardiomyopathy characterized by myocardial dysfunction of the left ventricle (both diastolic and posterior systolic), has been well studied in both humans and animals [57-59]. The mechanism of diabetic cardiac dysfunction is complex and involves an increase in oxidative / nitrosative stress [60-63], the activation of several later transcription factors, proinflammatory pathways and cell death as NF-אB , poly (ADP-ribose) polymerase (PARP) [64], and MAPKs $[65,66]$, inactivation of pro-survival pathways such as Akt [67], which ultimately culminates in cell death [68], changes in composition of the extracellular matrix with increased cardiac fibrosis and increased inflammation [21,65].

In addition, activation of the CB1 receptor in the cardiovascular 
system by endocannabinoids may also contribute to the pathophysiology of multiple cardiovascular diseases, including heart failure and atherosclerosis [69]. Unlike THC, the CBD, which has been approved for the treatment of inflammation, pain and spasticity associated with multiple sclerosis in humans since 2005 in Canada [70], it does not bind to these receptors [71], and therefore, it is devoid of psychoactive properties and has no potential to cause adverse cardiac toxicity [18]. Importantly, CBD is well tolerated without side effects when administered chronically to humans $[72,73]$. A previous study has shown cardiac protection by $\mathrm{CBD}$ in myocardial ischemic reperfusion injury [74]; with which, the possible protective effects of CBD in diabetic hearts and in primary human cardiomyocytes exposed to high glucose levels have been studied, finding a potential effect of CBD for the prevention and treatment of diabetic complications [21].

On the other hand, there are several risk factors for diabetic ketoacidosis in patients with DM1, including: myocardial infarction, stroke, pancreatitis, glucocorticoids, thiazide diuretics, sympathomimetics, and second generation antipsychotics and cocaine use, among others $[75,76]$. Psychological problems associated with eating disorders and deliberate omission of insulin, particularly in young patients with DM1, as well as a poor compliance with the insulin regimen are common precipitants.

Cannabis has been identified as another possible contributor to ketonemia; the dominant effect of cannabis with respect to the acid-base balance is the respiratory system with a change in respiratory rate and oxygen tension and not $\mathrm{pH}, 5$ but in these diabetic cannabis users, the near-normal or high $\mathrm{pH}$ would suggest an acute or chronic alcalemia. Clinical presentation with vomiting adds the possibility of an alteration of the compound acid base (with a component of metabolic alkalosis), but vomiting was rarely present in these patients and was equally present in acidotic patients. Ketosis requires intravenous insulin and fluid replacement and patients with an unexplained or unusual acid-base balance in diabetic ketosis or ketoacidosis should be evaluated for the use of illicit drugs, especially cannabis [76-79].

\section{Conclusions}

Despite the evidence presented, it is necessary to carry out longitudinal studies to determine the beneficial association between the use of marijuana and the treatment of diabetes and other nontransmissible chronic diseases. In Colombia, where marijuana is produced and consumed and where diabetes has high rates of prevalence, incidence and morbidity and mortality, this type of study could provide important information for the management of these pathologies that affect the quality of life of those who suffer.

\section{Acknowledgments}

The authors wish to acknowledge the financial support provided by the National Institute of Health of Colombia.

\section{References}

1. United Nations Office on Drugs and Crime. Sales No. E.15.
XI.6. United Nations Publication; 2016. World drug report 2015.

2. Thomas G, Kloner RA, Rezkalla S. Adverse cardiovascular, cerebrovascular, and peripheral vascular effects of marijuana inhalation: what cardiologists need to know. The American Journal of Cardiology. 2014; 113: 187-190.

3. Degenhardt L, Coffey C, Romaniuk H, et al. The persistence of the association between adolescent cannabis use and common mental disorders into young adulthood. Addiction. 2013; 108: 124-133.

4. Andréasson S, Engström A, Allebeck P, et al. Cannabis and schizophrenia. A Longitudinal Study of Swedish conscripts. The Lancet. 1987; 330: 1483-1486.

5. Zammit S, Allebeck P, Andreasson S, et al. Self-reported cannabis use as a risk factor for schizophrenia in Swedish conscripts of 1969: historical cohort study. British Medical Journal. 2002; 325: 1199-1201.

6. Lev-Ran S, Roerecke M, Le Foll B, et al. The association between cannabis use and depression: a systematic review and meta-analysis of longitudinal studies. Psychological Medicine. 2014; 44: 797-810.

7. Cox RG, Zhang L, Johnson WD, et al. Academic performance and substance use: findings from a state survey of public high school students. Journal of School Health. 2007; 77: 109-115.

8. Tetrault JM, Crothers K, Moore BA, et al. Effects of marijuana smoking on pulmonary function and respiratory complications: a systematic review. Archives of Internal Medicine. 2007; 167: 221-228.

9. Tashkin DP, Baldwin GC, Sarafian T, et al. Respiratory and immunologic consequences of marijuana smoking. Journal of Clinical Pharmacology. 2002; 42: 71S-81S.

10. Callaghan RC, Allebeck P, Sidorchuk A, et al. Marijuana use and risk of lung cancer: a 40-year cohort study. Cancer Causes and Control. 2013; 24: 1811-1820.

11. Hackam DG. Cannabis and stroke: systematic appraisal of case reports. Stroke. 2015; 46: 852-856.

12. Danielsson AK, Lundin A, Yaregal A, et al. Cannabis Use as Risk or Protection for Type 2 Diabetes: A Longitudinal Study of 18000 Swedish Men and Women. J Diabetes Res. 2016.

13. Tamayo T, Rosenbauer J, Wild SH, et al. Diabetes in Europe: an update. Diabetes Research and Clinical Practice. 2014; 103: 206-217.

14. Cullmann M, Hilding A, Östenson CG. Alcohol consumption and risk of pre-diabetes and type 2 diabetes development in a Swedish population. Diabetic Medicine. 2012; 29: 441-452.

15. Hilding A, Eriksson AK, Agardh EE, et al. The impact of family history of diabetes and lifestyle factors on abnormal glucose regulation in middle-aged Swedish men and women. Diabetologia. 2006; 49: 2589-2598.

16. World Health Organization (WHO). The Health and Social Effects of Nonmedical Cannabis Use. Geneva, Switzerland: World Health Organization (WHO); 2016.

17. Pacher P, Batkai S, Kunos G. The endocannabinoid system as an emerging target of pharmacotherapy. Pharmacol Rev. 2006; 58: 389-462.

18. Weiss L, Zeira M, Reich S, et al. Cannabidiol arrests onset 
of autoimmune diabetes in NOD mice. Neuropharmacology. 2008; 54: 244-249.

19. Izzo AA, Borrelli F, Capasso R, et al. Non-psychotropic plant cannabinoids: new therapeutic opportunities from an ancient herb. Trends Pharmacol Sci. 2009; 30: 515-527.

20. El-Remessy AB, Al-Shabrawey M, Khalifa Y, et al. Neuroprotective and blood-retinal barrier-preserving effects of cannabidiol in experimental diabetes. Am J Pathol. 2006; 168: 235-244.

21. Rajesh M, Mukhopadhyay P, Bátkai S, et al. Cannabidiol attenuates cardiac dysfunction, oxidative stress, fibrosis, inflammatory and cell death signaling pathways in diabetic cardiomyopathy. J Am Coll Cardiol. 2010; 56: 2115-2125.

22. Croxford JL, Yamamura T. Cannabinoids and the immune system: potential for the treatment of inflammatory diseases? J Neuroimmunol. 2005; 166: 3-18.

23. Lu D, Vemuri VK, Duclos RI Jr, Makriyannis A. The cannabinergic system as a target for anti-inflammatory therapies. Curr Top Med Chem. 2006; 6: 1401-1426.

24. Weiss L, Zeira M, Reich S, et al. Cannabidiol lowers incidence of diabetes in non-obese diabetic mice. Autoimmunity. 2006; 39: 143-151.

25. Wargent ET, Zaibi MS, Silvestri C, et al. The cannabinoid $\Delta(9)$-tetrahydrocannabivarin (THCV) ameliorates insulin sensitivity in two mouse models of obesity. Nutr Diabetes. 2013; 3: e68.

26. Kirkham TC. Cannabinoids and appetite: food craving and food pleasure. International Review of Psychiatry. 2009; 21: 163-171.

27. Di Marzo V, Piscitelli F, Mechoulam R. Cannabinoids and endocannabinoids in metabolic disorders with focus on diabetes. In: Schwanstecher M., editor. DiabetesPerspectives in Drug Therapy. 2011; 75-104.

28. Foltin RW, Fischman MW, Byrne MF. Effects of smoked marijuana on food intake and body weight of humans living in a residential laboratory. Appetite. 1988; 11: 1-14.

29. Smit E, Crespo CJ. Dietary intake and nutritional status of US adult marijuana users: results from the Third National Health and Nutrition Examination Survey. Public Health Nutr. 2001; 4: 781-786.

30. Rodondi N, Pletcher MJ, Liu K, et al. Marijuana use, diet, body mass index, and cardiovascular risk factors (from the CARDIA study). Am J Cardiol. 2006; 98: 478-484.

31. Le Strat Y, Le Foll B. Obesity and cannabis use: results from 2 representative national surveys. Am J Epidemiol. 2011; 174 : 929-933.

32. Rajavashisth TB, Shaheen M, Norris KC, et al. Decreased prevalence of diabetes in marijuana users: cross-sectional data from the National Health and Nutrition Examination Survey (NHANES) III. BMJ Open. 2012; 2: e000494.

33. Migrenne S, Lacombe A, Lefèvre AL, et al. Adiponectin is required to mediate rimonabant-induced improvement of insulin sensitivity but not body weight loss in diet-induced obese mice. Am J Physiol Regul Integr Comp Physiol. 2009; 296: R929-R935.

34. Sowers JR. Endocrine functions of adipose tissue: focus on adiponectin. Clin Cornerstone. 2008; 9: 32-38.

35. Wierzbicki A, Pendleton S, McMahon Z, et al. Rimonabant improves cholesterol, insulin resistance and markers of nonalcoholic fatty liver in morbidly obese patients: a retrospective cohort study. Int J Clin Pract. 2011; 65: 713-715.

36. Després JP, Golay A, Sjöström L. Effects of rimonabant on metabolic risk factors in overweight patients with dyslipidemia. N Engl J Med. 2005; 353: 2121-2134.

37. Levendal R, Schumann D, Donath M, et al. Cannabis exposure associated with weight reduction and b-cell protection in an obese rat model. Phytomedicine. 2012; 19: 575-582.

38. Ravinet-Trillou C, Delgorge $\mathrm{C}$, Menet $\mathrm{C}$, etal. CB1 cannabinoid receptor knockout in mice leads to leanness, resistance to dietinduced obesity and enhanced leptin sensitivity. Int J Obes. 2004; 28: 640-648.

39. Penner EA, Buettner H, Mittleman MA. The impact of marijuana use on glucose, insulin, and insulin resistance among US adults. The American Journal of Medicine. 2013; 126: 583-589.

40. Jamshidi N, Taylor DA. Anandamide administration into the ventromedial hypothalamus stimulates appetite in rats. British Journal of Pharmacology. 2001; 134: 1151-1154.

41. Williams CM, Kirkham TC. Anandamide induces overeating: mediation by central cannabinoid (CB1) receptors. Psychopharmacology. 1999; 143: 315-317.

42. Ngueta G, Bélanger RE, Laouan-Sidi EA, et al. Cannabis use in relation to obesity and insulin resistance in the inuit population. Obesity. 2015; 23: 290-295.

43. Alshaarawy $\mathrm{O}$, Anthony JC. Cannabis smoking and diabetes mellitus: results from meta-analysis with eight independent replication samples. Epidemiology. 2015; 26: 597-600.

44. Rajavashisth TB, Shaheen M, Norris KC, et al. Decreased prevalence of diabetes in marijuana users: cross-sectional data from the National Health and Nutrition Examination Survey (NHANES) III. BMJ Open. 2012; 2: e000494.

45. Sidney S. Marijuana Use and Type 2 Diabetes Mellitus: a Review, Curr Diab Rep. 2016; 16: 117.

46. Dagogo-Jack S. Diabetes, Risk of Diabetes and Nonprescription Drugs: Mechanisms and Approaches to Risk Reduction, Alexandria: American Diabetes Association. 2016.

47. Bancks MP, Pletcher MJ, Kertesz SG, et al. Marijuana use and risk of prediabetes and diabetes by middle adulthood: the Coronary Artery Risk Development in Young Adults (CARDIA) study, Diabetology. 2015; 58: 2736-2744.

48. Comelli F, Bettoni I, Colleoni M, et al. Beneficial Effects of a Cannabis sativa Extract Treatment on Diabetes-induced Neuropathy and Oxidative Stress, Phytother Res. 2009; 23: 1678-1684.

49. Selvarajah D, Gandhi R, Emery CJ, et al. Randomized placebocontrolled double-blind clinical trial of cannabis-based medicinal product (Sativex) in painful diabetic neuropathy: depression is a major confounding factor. Diabetes Care. 2010; 33:128-130.

50. Wallace MS, Marcotte TD, Umlauf A, et al. Efficacy of inhaled cannabis on painful diabetic neuropathy J Pain. 2015; 16: 616-627. 
51. Doğrul A, Gül H, Yildiz O, et al. Cannabinoids blocks tactile allodynia in diabetic mice without attenuation of its antinociceptive effect. Neurosci Lett. 2004; 368: 82-86.

52. Ulugol A, Karadag HC, Ipci Y, et al. The effect of WIN 55,2122 , a cannabinoid agonist, on tactile allodynia in diabetic rats. Neurosci Lett. 2004; 371: 167-170.

53. Alshaarawy O, Anthony JC. Cannabis smoking and diabetes mellitus: results from meta-analysis with eight independent replication samples, Epidemiology. 2015; 26: 597-600.

54. Malfait AM, Gallily R, Sumariwalla PF, et al. The nonpsychoactive cannabis constituent cannabidiol is an oral anti-arthritic therapeutic in murine collageninduced arthritis. Proc Natl Acad Sci USA. 2000; 97: 9561-9566.

55. Rajesh M, Mukhopadhyay P, Bátkai S, et al. Cannabidiol attenuates high glucoseinduce endothelial cell inflammatory response and barrier disruption. Am J Physiol Heart Circ. Physiol. 2007; 293: H610-H619.

56. Zuardi AW. Cannabidiol: from an inactive cannabinoid to a drug with wide spectrum of action. Rev Bras Psiquiatr. 2008; 30: 271-280.

57. Fein FS. Diabetic cardiomyopathy. Diabetes Care. 1990;13: 1169-1179.

58. Regan TJ, Ahmed S, Haider B, et al. Diabetic cardiomyopathy: experimental and clinical observations. N J Med. 1994; 91: 776-778.

59. Asbun J, Villarreal FJ. The pathogenesis of myocardial fibrosis in the setting of diabetic cardiomyopathy. J Am Coll Cardiol. 2006; 47: 693-700.

60. Kajstura J, Fiordaliso F, Andreoli AM, et al. IGF1 overexpression inhibits the development of diabetic cardiomyopathy and angiotensin II-mediated oxidative stress. Diabetes. 2001; 50: 1414-1424.

61. Pacher P, Beckman JS, Liaudet L. Nitric oxide and peroxynitrite in health and disease. Physiol Rev. 2007; 87: 315-424.

62. Cai L, Wang Y, Zhou G, et al. Attenuation by metallothionein of early cardiac cell death via suppression of mitochondrial oxidative stress results in a prevention of diabetic cardiomyopathy. J Am Coll Cardiol. 2006; 48: 1688-1697.

63. Wang Y, Feng W, Xue W, et al. Inactivation of GSK-3 beta by metallothionein prevents diabetes-related changes in cardiac energy metabolism, inflammation, nitrosative damage, and remodeling. Diabetes. 2009; 58: 1391-1402.

64. Pacher P, Liaudet L, Soriano FG, et al. The role of poly (ADP-ribose) polymerase activation in the development of myocardial and endothelial dysfunction in diabetes. Diabetes. 2002; 51: 514-521.

65. Westermann D, Rutschow S, Van Linthout S, et al. Inhibition of p38 mitogen-activated protein kinase attenuates left ventricular dysfunction by mediating pro-inflammatory cardiac cytokine levels in a mouse model of diabetes mellitus. Diabetologia. 2006; 49: 2507-2513.

66. Thandavarayan RA, Watanabe K, Ma M, et al. Dominantnegative p38 \{alpha\} mitogen-activated protein kinase prevents cardiac apoptosis and remodeling after streptozotocininduced diabetes mellitus. Am J Physiol Heart Circ Physiol. 2009; 297: H911-H919.

67. Van Linthout S, Spillmann F, Riad A, et al. Human Apolipoprotein A-I Gene Transfer Reduces the Development of Experimental Diabetic Cardiomyopathy. Circulation. 2008; 117: 1563-1573.

68. Frustaci A, Kajstura J, Chimenti C, et al. Myocardial cell death in human diabetes. Circ Res. 2000; 87: 1123-1132.

69. Pacher P, Steffens S. The emerging role of the endocannabinoid system in cardiovascular disease. Semin Immunopathol. 2009; 31: 63-77.

70. Barnes MP. Sativex: clinical efficacy and tolerability in the treatment of symptoms of multiple sclerosis and neuropathic pain. Expert Opin Pharmacother. 2006; 7: 607-615.

71. Thomas BF, Gilliam AF, Burch DF, et al. Comparative receptor binding analyses of cannabinoid agonists and antagonists. J Pharmacol Exp Ther. 1998; 285: 285-292.

72. Cunha JM, Carlini EA, Pereira AE, et al. Chronic administration of cannabidiol to healthy volunteers and epileptic patients. Pharmacology. 1980; 21: 175-185.

73. Consroe P, Laguna J, Allender J, et al. Controlled clinical trial of cannabidiol in Huntington's disease. Pharmacol Biochem Behav. 1991; 40: 701-708.

74. Durst R, Danenberg H, Gallily R, et al. Cannabidiol, a nonpsychoactive Cannabis constituent, protects against myocardial ischemic reperfusion injury. Am J Physiol Heart Circ Physiol. 2007; 293: H3602-H3607.

75. Warner EA, Greene GS, Buchsbaum MS, et al. Diabetic ketoacidosis associated with cocaine use. Arch Intern Med. 1998; 158: 1799-1802.

76. Hennessy A. Cannabis masks diabetic ketoacidosis. BMJ Case Rep. 2011.

77. Nyenwe EA, Loganathan RS, Blum S, et al. Active use of cocaine: an independent risk factor for recurrent diabetic ketoacidosis in a city hospital. Endocr Pract. 2007; 13: 22-29.

78. Ng RS, Darko DA, Hillson RM. Street drug use among young patients with Type 1 diabetes in the UK. Diabet Med. 2004; 21: 295-296.

79. Greco AV, Bertoli A, Caputo S, et al. Ketoalkalosis as a result of triple derangement of acid-base equilibrium in a diabetic patient. Acta Diabetol Lat. 1985; 22: 73-77. 